\title{
Time-Domain Simulation of Directive Sources in 3-D Digital Waveguide Mesh-Based Acoustical Models
}

\author{
Hüseyin Hacıhabiboğlu, Member, IEEE, Banu Günel, Member, IEEE, and Ahmet M. Kondoz, Member, IEEE
}

\begin{abstract}
Digital waveguide mesh (DWM) models offer a simple, accurate, time-domain, numerical solution of the wave equation. A specific case where such accurate and computationally simple solutions are needed is the acoustical modeling of open or closed volumes. It is possible to model 3-D propagation of waves in enclosures such as rooms using DWM models. Generally, idealized omnidirectional sources are used for obtaining the impulse response in the DWM. However, real-life sound sources are never completely isotropic, causing wavefronts with directional properties. This paper presents two methods to simulate analytical and empirical directivities in 3-D DWM models in the far-field. The first method is based on the direct excitation of the mesh with the velocity component of the directional source and is used to simulate analytical sources. The second method is based on the weighting of velocity components generated by an omnidirectional source at different octave-bands and is used to simulate sources with frequency-dependent empirical directivity functions. A simple interpolation method for obtaining a closed-form description of the directivity function from incomplete directivity data is also proposed. Simulation results are presented for two sources in an acoustical model of a rectangular room.
\end{abstract}

Index Terms-Acoustic modeling, digital waveguide mesh (DWM), multidimensional wave propagation, sound source directivity.

\section{INTRODUCTION}

$\mathbf{T}$ HE PREDICTION of sound fields inside open or closed acoustical volumes such as city canyons or rooms has been a traditional research topic with important applications in audio and acoustical signal processing. The research in the area has been driven by the need for the modeling and simulation of room acoustics and the prediction of environmental noise. Efforts have mainly been concentrated on methods employing the principles of geometrical acoustics, largely due to their computational simplicity. Such methods include ray tracing [1] and its derivatives [2], and image-source method (ISM) [3] among others. While these methods have significant practical value, they are hindered by several inherent problems in accounting for certain properties of wave related phenomena

Manuscript received August 6, 2007; revised February 11, 2008. This work was supported by VISNET II, a European Network of Excellence (http://www. visnetnoe.org), funded under the European Commission IST FP6 program. The work reported in this article was carried out while H. Hacihabiboğlu was with the University of Surrey. The associate editor coordinating the review of this manuscript and approving it for publication was Prof. Vesa Välimäki.

H. Hacihabiboğlu is with the Centre for Digital Signal Processing Research (CDSPR), Division of Engineering, King's College London, London WC2R 2LS, U.K. (e-mail: huseyin.hacihabiboglu@ @cl.ac.uk).

B. Günel and A. M. Kondoz are with the I-Lab, Centre for Communication Systems Research (CCSR), University of Surrey, Guildford GU2 7XH, U.K. (e-mail: b.gunel@surrey.ac.uk; a.kondoz@surrey.ac.uk).

Digital Object Identifier 10.1109/TASL.2008.921759 such as diffraction and occlusion. The simulation of these phenomena requires complex modifications to the original methods [4]. In addition, they also fail to provide accurate predictions at low and midrange frequencies.

Analytical solutions of the wave equation for volumes with complex geometries are not possible. However, numerical modeling methods which utilize approximate local solutions of the wave equation provide accurate predictions of the sound field at a given problem domain. The problem domain is discretized into a finite number of elements and the numerical solution of the wave equation is obtained by basic algebraic operations either in time or in frequency domains. Several different numerical methods exist such as the finite-element method (FEM) [5], boundary element method (BEM) [6], finite-difference time-domain (FDTD) methods [7], [8], wave digital filters (WDF) [9], and digital waveguide meshes (DWMs) [10] which make it possible to obtain accurate predictions of sound fields at low and midrange frequencies. As these methods are based on the solution of the wave equation, wave-related phenomena such as diffraction and occlusion require no additional computational effort. Another advantage of such numerical methods is that unlike geometrical modeling methods, they provide impulse responses at each and every discrete space element without the need to explicitly address visibility or obstruction issues which make up for the most of the computational load in geometrical modeling methods [11]. DWMs, among other numerical methods, provide a computationally simple, time-domain approach to numerical modeling of sound fields in volumes. DWMs provide the traveling-wave solution for wave propagation at low and midrange frequencies. DWMs were also shown to provide equivalent results with the more mainstream FDTD methods [12].

A common problem in all acoustical prediction methods is the modeling of the source directivity. Usually, impulse responses in a modeled acoustical system are obtained by using an omnidirectional point source excitation, and by registering the pressure fluctuation at a given point in the modeled volume. However, acoustical sources are never omnidirectional in real life. All real sound sources such as the human mouth [13]-[15] or musical instruments [16] radiate sound unevenly towards different directions and for different frequencies. Geometrical modeling methods try to solve this problem by directionally weighting the emitted rays or beams in ray or beam tracing methods [17] or directionally filtering the impulse responses of individual image sources in ISM [18].

There exist several methods for numerically simulating acoustical fields generated by directional sound sources based on the source simulation concept [19]. These include spherical harmonic decomposition [20], wave superposition [21], [22], 
and multipole synthesis [20], [23]. These methods are based on the harmonic solution of the wave equation and they operate in the frequency domain. As such, they generally provide a correct simulation of the acoustical field generated by a directional source at a single frequency per simulation and not for a broadband excitation. A time-domain formulation of the source simulation method for broadband excitation has also been proposed [24].

There has been little emphasis on the simulation of directional acoustical sources with time-domain methods such as DWM-based acoustical models. A method for the simulation of sources with frequency-independent directivity functions on 2-D DWMs has previously been proposed by the authors [25]. Subsequently, the direct application of the method of multipole synthesis has been proposed as an alternative solution to simulate source directivity for the harmonic excitation of time-domain numerical acoustical models [26]. Both methods are only partly useful in the sense that the former cannot simulate frequency-dependent source directivity, and the latter in practice works only for single or multiple excitation frequencies which makes it impossible to simulate the model with a broadband, impulsive stimulus covering the whole spectrum.

This paper presents two methods for the simulation of sources with 1) analytically defined, and 2) empirically measured directivity functions within 3-D DWM-based models. It is organized as follows. Section II presents a brief overview of digital waveguide meshes. Section III provides a physical interpretation of the notion of source directivity. Section IV proposes two methods for the simulation of analytical and empirical sources in 3-D DWM models. In addition, an interpolation method for obtaining a closed form directivity function from incomplete directivity measurements is presented. Section $\mathrm{V}$ provides examples of simulation on a 3-D DWM mesh using source models for an ideal dipole and a baritone singer. Section VI concludes the paper.

\section{DWM MODELS}

DWMs provide second-order accurate solutions of the wave equation in the discrete-time domain. DWMs employ a traveling wave solution and can easily be integrated with other signal processing algorithms that are involved in audio synthesis and acoustical modeling. DWM models are generalizations to multiple dimensions of the concept of digital waveguides which have been used to simulate acoustical resonators such as vibrating strings [10], [27]. They have successfully been used in a number of acoustical modeling problems such as the modeling of the vocal tract [28], drum membranes [29], and room acoustics [30]-[32]. There are two types of DWM models, the W-DWM which employs wave variables and the K-DWM which employs Kirchhoff variables [33], [34]. The DWM model employed in this paper is the W-DWM, which is the original formulation of the DWM.

DWM models require the spatial discretization of the problem domain into a finite number of elements called the scattering junctions. Each scattering junction is connected to its neighbors via digital waveguides which are simple bidirectional integer delay elements forming a mesh [35]. The junction equations determine how scattering is carried out at the junction.
Let us consider a scattering junction $\left(S_{\mathbf{x}}\right)$ positioned at the point $\mathbf{x}=\left[\begin{array}{lll}x_{0} & \cdots & x_{K-1}\end{array}\right]$ in a $K$-dimensional discretized problem domain. $S_{\mathrm{x}}$ is connected to a number of other scattering junctions via bidirectional delays over their input and output ports. Pressure values at each port $\left(p_{i}\right)$ and the scattering junction pressure $\left(p_{J}\right)$ are equal to each other, and the sum of all port velocities $\left(v_{i}\right)$ of the given scattering junction is zero. If the scattering junction has $L$ ports this can be expressed as

$$
\begin{aligned}
p_{1} & =p_{2}=\ldots=p_{L}=p_{J} \\
\sum_{i=1}^{L} v_{i} & =0
\end{aligned}
$$

The flow-like wave variables, $p_{i}^{-}$and $p_{i}^{+}$, whose difference correspond to particle velocity at port $i, \mathbf{v}_{i}$ are defined as

$$
\begin{aligned}
\mathbf{v}_{i} & =Y_{i}\left(p_{i}^{-}-p_{i}^{+}\right) \hat{\mathbf{u}}_{i} \\
p_{J} & =p_{i}^{-}+p_{i}^{+} .
\end{aligned}
$$

Here, $p_{i}^{+}$and $p_{i}^{-}$are the incoming (incident) and outgoing (reflected) wave variables at the port $i$, respectively, $Y_{i}$ is the port admittance, and $\hat{\mathbf{u}}_{i}$ represents the outward radial unit vector from the junction along the direction of the corresponding waveguide. The junction pressure is then defined as a function of incoming pressure wave variables as

$$
p_{J}=2 \sum_{i=1}^{L} Y_{i} p_{i}^{+} / \sum_{i=1}^{L} Y_{i} .
$$

For isotropic media, all port admittances are equal, and it is possible to express junction pressure as a weighted sum of incoming wave variables such that

$$
p_{J}=\frac{2}{L} \sum_{i=1}^{L} p_{i}^{+} .
$$

Once the junction variables are obtained from incoming wave variables using (6), the outgoing wave variables can be calculated using (4) and propagated to the junction's corresponding neighbors as their incoming wave variables for the next iteration step. As a result, the DWM model provides the traveling wave solution of the wave equation at each iteration for discrete time and space.

Therefore, it is possible to define the operation of the DWM as a two step process.

1) Scattering pass is the calculation of the outgoing wave variables carried out by scattering the incoming wave variables in a junction using (4) and (6). It is possible to skip the calculation of the junction pressure and use a matrix multiplication instead to obtain the outgoing wave variables directly from the incoming wave variables [36].

2) Propagation step is the shifting of outgoing wave variables through the bidirectional delays (i.e., digital waveguides) within the DWM to obtain the incoming wave variables for the next iteration.

The upper frequency limit of the DWM mesh is given as $f_{u}=$ $0.25 f_{S}$, where $f_{S}$ is the update frequency of the mesh [31]. Above this frequency limit, the simulation results will not be 
valid due to aliasing. The actual frequency bandwidths of multidimensional DWMs are direction dependent. However, they are always less than $f_{u}$.

Several different 3-D mesh topologies exist for simulating wave propagation in volumes [37], [38]. Among these, the uniform rectilinear mesh topology provides a more attractive option in terms of the intuitive positioning of its junctions and the accuracy of numerical derivatives when the mesh density (i.e., the number of junctions per unit volume) is constant [39]. It also allows a more structured memory organization and has a well-documented prospect for software optimization and parallelization [40], [41]. Therefore, although it has higher directional dispersion errors [42] and a higher computational complexity [38] than other 3-D mesh topologies, uniformly sampled rectilinear topology is preferred for the examples in this paper. It should be noted that the methods proposed in this paper can be applied to other mesh topologies with slight modifications.

A given junction in a uniformly sampled rectilinear mesh has a total of six neighboring junctions on its $x, y$, and $z$ axes. As a matter of convenience, the junctions can be indexed consistently with their Cartesian coordinates. The ports of a junction associated with each neighboring junction can be geographically named as N (North), S (South), E (East), W (West), U (Up), and $\mathrm{D}$ (Down) situated at $\mathbf{x}_{N}=\mathbf{x}+[0 \Delta d 0], \mathbf{x}_{S}=\mathbf{x}-[0 \Delta d 0]$, $\mathbf{x}_{E}=\mathbf{x}+[\Delta d 00], \mathbf{x}_{W}=\mathbf{x}-[\Delta d 00], \mathbf{x}_{U}=\mathbf{x}+\left[\begin{array}{lll}0 & 0 & \Delta d\end{array}\right]$, and $\mathbf{x}_{D}=\mathbf{x}-\left[\begin{array}{lll}0 & 0 & \Delta d\end{array}\right]$, respectively. Here, $\Delta d$ represents the unit space between the junctions in the DWM or its spatial sampling period. The wave speed $c^{\prime}$ in uniform rectilinear 3-D DWM is defined as

$$
c^{\prime}=\frac{f_{S} \Delta d}{\sqrt{3}}
$$

The spatial sampling period $\Delta d$ is selected such that $c^{\prime}$ is equal to the speed of sound in air at the given temporal sampling frequency.

The readers are referred to [10], [12], [35], [36], and [42] for further details of the DWM models including their stability, dispersion, mesh topologies, and implementation.

\section{SOURCE DIRECTIVITY}

The source directivity $\Gamma(\theta, \phi)$ is intimately related to the $r a$ diation pattern of a source $J(\theta, \phi)$ defined as the acoustic power radiated per unit solid angle [43], where $0 \leq \theta<2 \pi$ and $-\pi / 2 \leq \phi \leq \pi / 2$ are the azimuth and elevation angles, respectively. Let us assume for the time being that the radiation pattern, and thus the directivity function, are independent of frequency.

The instantaneous intensity of the sound field in the radial direction from the center of the directional source is defined as $\mathbf{I}=p \mathbf{v}$, where $p$ is the instantaneous pressure and $\mathbf{v}$ is the instantaneous particle velocity component of the sound field. The average intensity at a radial distance $r$ is defined as

$$
\begin{aligned}
\overline{\mathbf{I}}(r, \theta, \phi) & =\frac{J(\theta, \phi)}{r^{2}} \hat{\mathbf{u}}_{r} \\
& =\frac{1}{\Delta T} \int_{t_{0}}^{t_{0}+\Delta T} \mathbf{I}(r, \theta, \phi, t) d t
\end{aligned}
$$

where $\hat{\mathbf{u}}_{r}$ is the unit vector in the radial direction of the source. Then, the radiation pattern of the source, $J(\theta, \phi)$, can be expressed as

$$
J(\theta, \phi)=\frac{r^{2}}{\Delta T} \int_{t_{0}}^{t_{0}+\Delta T} p \mathbf{v} \cdot \hat{\mathbf{u}}_{r} d t
$$

Let us consider the velocity potential, $\Phi$, from which the pressure and velocity parameters can be derived, such that

$$
\mathbf{v}=\nabla \Phi, \quad p=-\rho \frac{\partial \Phi}{\partial t}
$$

where $\rho$ represents the ambient density, and $\nabla$ denotes the gradient operator.

The velocity potential for an omnidirectional, spherically symmetric source is $\Phi_{o}(r, t)=-G\left(t-c^{-1} r\right) / \rho r$, where $G$ is an arbitrary function. It is possible to express the omnidirectional velocity and pressure components as [43]

$$
\begin{aligned}
& \mathbf{v}_{o}(r, t)=\left[\frac{p_{o}(r, t)}{c \rho}+\frac{G\left(t-c^{-1} r\right)}{\rho r^{2}}\right] \hat{\mathbf{u}}_{r} \\
& p_{o}(r, t)=\frac{1}{r} \frac{\partial}{\partial t} G\left(t-c^{-1} r\right) .
\end{aligned}
$$

The second term on the right-hand side of (12) is inversely proportional to the square of distance from the source and is negligible in the far-field. The radiation pattern in the far-field for an omnidirectional source can thus be defined as

$$
J_{o}=\frac{1}{\rho c \Delta T} \int_{t_{0}}^{t_{0}+\Delta T} g^{2}\left(t-c^{-1} r\right) d t
$$

where $g(t)=d G(t) / d t$. The omnidirectional radiation pattern $J_{o}$ is constant across $\theta$ and $\phi$ as the source has equal intensity for all directions.

Let us define a directional velocity potential $\Phi_{d}$ such that

$$
\Phi_{d}(r, \theta, \phi, t)=\Gamma(\theta, \phi) \Phi_{o}(r, t)
$$

where the omnidirectional velocity potential $\Phi_{O}$ is weighted by the directivity function $\Gamma(\theta, \phi)$. At an observation point in the acoustical far-field of the source and with the constant vorticity assumption [43], the directional velocity and pressure components can be expressed using (11) as

$$
\begin{aligned}
& \mathbf{v}_{d}(r, \theta, \phi, t)=\Gamma(\theta, \phi) \mathbf{v}_{o}(r, t) \\
& p_{d}(r, \theta, \phi, t)=\Gamma(\theta, \phi) p_{o}(r, t) .
\end{aligned}
$$

Then, the radiation pattern as measured in the far-field for the directional source is

$$
J_{d}(\theta, \phi)=|\Gamma(\theta, \phi)|^{2} J_{o} .
$$

Using the concept of secondary sources on the wavefront, following the Huygens' principle and the Kirchhoff-Helmholtz integral, it may be suggested from (16) and (17) that, if the omnidirectional velocity field or pressure field and the source directivity function at a measurement distance $R_{0}$ in the acoustical far-field are known, the directional response of the source can be obtained for $t \geq c^{-1} R_{0}$. 
Following the discussion on the far-field component, it is possible to define two different directivity functions: Analytical directivity function refers to the directivity function mathematically defined for the source at the instant of excitation. Empirical directivity function, in contrast, is the directivity function measured at a distance, generally in the acoustical far-field, and is thus considered to be free from the contribution of the near-field components.

The discussion above has two important results which will be discussed further in the following section.

1) If the analytical far-field directivity function is known for a sound source at the instant of excitation, it is possible to excite the DWM model using the velocity field derived from an omnidirectional velocity potential by directional weighting to simulate the wave propagation from the source. The simulated wavefront will display the properties of that source in the far-field.

2) If the empirical directivity function of a sound source measured at the acoustical far-field is known, it may be used to obtain a directional velocity vector field to simulate wave propagation for distances greater than or equal to the distance at which the empirical directivity function measurement was made.

\section{Simulation of Source Directivity ON 3-D DWM MODELS}

As mentioned above, directivity functions are classified into two groups in this article as analytical and empirical directivity functions. It is possible to use analytical directivity functions to weight the initial velocity field directly for designating a directional source distribution to excite the DWM model. The empirical directivity functions are valid only for distances greater than or equal to the distance at which the measurement is made. Therefore, sources with known analytical and empirical directivity functions require different excitation strategies. Two methods are presented in this section. The first method is a generalization of the previous work by the authors [25] to three dimensions for the simulation of analytical source directivities. The second method is for the broadband simulation of sources with frequency-dependent empirical directivity functions.

\section{A. Analytical Source Directivity}

For obtaining the acoustical impulse response in a volume, DWM models are generally excited with a bandlimited spatial impulse by setting the junction pressures in such a way that the pressure values are symmetrically distributed around a central excitation point. After setting the initial junction pressure distribution, the iteration is started at the scattering step. If all the incoming wave variables are set to zero, all the outgoing wave variables will be equal to the initial junction pressure from (6) effectively designating each junction as an individual omnidirectional source. This allows for a bandlimited omnidirectional impulse to be positioned at the excitation point.

The analytical directivity function can be used for directionally weighting the source velocity distribution at the initial excitation stage of the DWM model. Instead of setting both the junction pressures and the incoming wave variables, the outgoing wave variables can be initialized and the iteration started with the propagation step instead of the scattering pass. In what follows, the simulation of directive sources using this strategy is described.

First, a spatially bandlimited excitation pulse is selected as the omnidirectional velocity potential. A good omnidirectional velocity potential function must be spherically symmetric and should provide a good attenuation at higher spatial frequencies to have a low spatial aliasing. A suitable candidate is the symmetric trivariate Gaussian function weighted with the inverse of the ambient density $\rho^{-1}$. This function can be expressed in the Cartesian coordinates for an arbitrary position $\mathbf{x}=[x y z]^{\mathbf{T}}$ and a mean spatial position $\bar{\mu}=\left[\mu_{x} \mu_{y} \mu_{z}\right]^{\mathbf{T}}$ as

$$
\Phi_{o}(\mathbf{x}, \bar{\mu}, n)=-\frac{1}{\rho(2 \pi)^{\frac{3}{2}} \sigma^{3}} e^{-|\mathbf{x}-\bar{\mu}|^{2} / 2 \sigma^{2}} \delta[n]
$$

where $\delta[n]$ is the Dirac delta function, $|\mathbf{x}|$ represents the $L^{2}$-norm of the vector $\mathbf{x}$. As the distribution is symmetric, all the variance terms are equal to $\sigma^{2}$ and all the covariance terms are zero. Note that, in order to designate $\Phi_{o}$ as the initial velocity potential of a sound source, its sign is selected to be negative.

The initial omnidirectional velocity, $\mathbf{v}_{o}(\mathbf{x}, n)=$ $\left[v_{x}(\mathbf{x}, n) v_{y}(\mathbf{x}, n) v_{z}(\mathbf{x}, n)\right]$, at the given position $\mathbf{x}$ can then be calculated using (11) with a change of coordinates from spherical to Cartesian as

$$
\mathbf{v}_{o}(\mathbf{x}, n)=\frac{e^{-|\mathbf{x}-\bar{\mu}|^{2} / 2 \sigma^{2}}}{\rho(2 \pi)^{\frac{3}{2}} \sigma^{5}}(\mathbf{x}-\bar{\mu}) \delta[n] .
$$

The directional velocity vectors with which the DWM is initialized can then be obtained as

$$
\tilde{\mathbf{v}}(\mathbf{x}, 0)=\Gamma(\theta-\vartheta, \phi-\varphi) \mathbf{v}_{o}(\mathbf{x}, 0)
$$

where $\Gamma(\theta, \phi)$ is the frequency-independent directivity function with arbitrary rotation angles $\vartheta$ in azimuth and $\varphi$ in elevation. Here, $\theta=\tan ^{-1}\left(\left(y-\mu_{y}\right) /\left(x-\mu_{x}\right)\right)$ and $\phi=\tan ^{-1}((z-$ $\left.\left.\mu_{z}\right) / \sqrt{\left(x-\mu_{x}\right)^{2}+\left(y-\mu_{y}\right)^{2}}\right)$.

The excitation of a DWM using such a directional velocity field requires setting the relevant parameters of the model accordingly. Due to the zero initial state assumption the incoming pressure variables for each junction are set to zero (i.e., $\left.p_{i}^{+}(\hat{\mathbf{x}}, 0)=0\right)$. The local velocity variables at each port can then be expressed in terms of the wave variables using (3) as

$$
\mathbf{v}_{i}(\hat{\mathbf{x}}, n)=Y p_{i}^{-}(\hat{\mathbf{x}}, n) \hat{\mathbf{u}}_{i}
$$

where $Y$ is the common port admittance, $i$ represents any of $\mathrm{N}, \mathrm{S}, \mathrm{E}, \mathrm{W}, \mathrm{U}$, or $\mathrm{D}$, and $\hat{\mathbf{x}}=[\hat{x} \hat{y} \hat{z}]^{\mathbf{T}}$ is the discrete spatial position in the model. In the original formulation of DWMs, a particle velocity variable is not defined at a junction. Therefore, we define the junction velocity at a given sample time $n$ as

$$
\begin{aligned}
& v_{x}(\hat{\mathbf{x}}, n)=v_{E}(\hat{\mathbf{x}}, n)-v_{W}(\hat{\mathbf{x}}, n) \\
& v_{y}(\hat{\mathbf{x}}, n)=v_{N}(\hat{\mathbf{x}}, n)-v_{S}(\hat{\mathbf{x}}, n) \\
& v_{z}(\hat{\mathbf{x}}, n)=v_{U}(\hat{\mathbf{x}}, n)-v_{D}(\hat{\mathbf{x}}, n)
\end{aligned}
$$

consistently with the Cartesian coordinate system. A similar approach for obtaining the particle velocity at a given junction has 
previously been proposed in a different context for modeling direction dependent absorption at DWM model boundaries [44].

At the initialization stage (i.e., $n=0$ ), the velocity components are

$$
\begin{aligned}
& \tilde{v}_{x}(\hat{\mathbf{x}}, 0)=Y\left[p_{E}^{-}(\hat{\mathbf{x}}, 0)-p_{W}^{-}(\hat{\mathbf{x}}, 0)\right] \\
& \tilde{v}_{y}(\hat{\mathbf{x}}, 0)=Y\left[p_{N}^{-}(\hat{\mathbf{x}}, 0)-p_{S}^{-}(\hat{\mathbf{x}}, 0)\right] \\
& \tilde{v}_{z}(\hat{\mathbf{x}}, 0)=Y\left[p_{U}^{-}(\hat{\mathbf{x}}, 0)-p_{D}^{-}(\hat{\mathbf{x}}, 0)\right] .
\end{aligned}
$$

Here, the assumption is made that the discretization of space is made in such a way that the local (i.e., junction) and global (i.e., model) coordinate systems are codirectional. In other words, the $x, y$, and $z$ directions for a given junction coincide with those of the overall model. The following selection is made for convenience: In octant I, $p_{W}^{-}(\hat{\mathbf{x}}, 0)=p_{S}^{-}(\hat{\mathbf{x}}, 0)=p_{D}^{-}(\hat{\mathbf{x}}, 0)=0$; in octant II, $p_{E}^{-}(\hat{\mathbf{x}}, 0)=p_{S}^{-}(\hat{\mathbf{x}}, 0)=p_{D}^{-}(\hat{\mathbf{x}}, 0)=0$; in octant III, $p_{E}^{-}(\hat{\mathbf{x}}, 0)=p_{N}^{-}(\hat{\mathbf{x}}, 0)=p_{D}^{-}(\hat{\mathbf{x}}, 0)=0$; in octant IV, $p_{W}^{-}(\hat{\mathbf{x}}, 0)=p_{N}^{-}(\hat{\mathbf{x}}, 0)=p_{D}^{-}(\hat{\mathbf{x}}, 0)=0$; in octant $\mathrm{V}, p_{W}^{-}(\hat{\mathbf{x}}, 0)=p_{S}^{-}(\hat{\mathbf{x}}, 0)=p_{U}^{-}(\hat{\mathbf{x}}, 0)=0$; in octant $\mathrm{VI}$, $p_{E}^{-}(\hat{\mathbf{x}}, 0)=p_{S}^{-}(\hat{\mathbf{x}}, 0)=p_{U}^{-}(\hat{\mathbf{x}}, 0)=0$; in octant VII, $p_{E}^{-}(\hat{\mathbf{x}}, 0)=p_{N}^{-}(\hat{\mathbf{x}}, 0)=p_{U}^{-}(\hat{\mathbf{x}}, 0)=0$; and finally in octant VIII, $p_{W}^{-}(\hat{\mathbf{x}}, 0)=p_{N}^{-}(\hat{\mathbf{x}}, 0)=p_{U}^{-}(\hat{\mathbf{x}}, 0)=0$.

The outgoing wave variables are then initialized accordingly by using the velocity values obtained by weighting the gradient of the selected velocity potential function defined as in (20) and (21). After the initialization stage, the iteration is started at the propagation step instead of the scattering pass. The wave propagation pattern and the room impulse response obtained at any given point in the far-field of the source is that of an excitation by the analytical directional source. It is possible to simulate simple sources such as ideal dipoles and quadrupoles as well as sources with analytically defined directivities such as line arrays [45] this way. Algorithm 1 provides a summary of the procedure described in this section.

\section{Algorithm 1 Analytical directivity}

Initialize ROOM \{ROOM is the 3-D DWM model\}.

Select an omnidirectional velocity potential $\Phi_{o}$.

Sample the velocity potential at the discrete positions in ROOM.

Obtain an omnidirectional velocity field $\mathbf{v}_{o}$ by the numerical gradient of sampled velocity potential.

Set the excitation point as $\bar{\mu}$.

\section{for all junctions in ROOM do}

Obtain the directive particle velocity at $\hat{\mathbf{x}}$ by weighting with $\Gamma(\theta, \phi)$.

The point $\hat{\mathbf{x}}$ in situated in OCTANT.

\section{if OCTANT is I then.}

$$
\begin{aligned}
& \text { Set } p_{E}^{-}(\hat{\mathbf{x}}, 0)=v_{x}(\hat{\mathbf{x}}, 0) / Y \\
& \text { Set } p_{N}^{-}(\hat{\mathbf{x}}, 0)=v_{y}(\hat{\mathbf{x}}, 0) / Y \\
& \text { Set } p_{U}^{-}(\hat{\mathbf{x}}, 0)=v_{z}(\hat{\mathbf{x}}, 0) / Y
\end{aligned}
$$

else if OCTANT is II then

\section{end if}

\section{end for}

repeat

$$
\text { propagate(ROOM) }
$$

scatter(ROOM)

until the desired iteration count is reached.

It should be noted that, due to the original assumption in (16) and (17) which decouples the azimuth $(\theta)$ and elevation $(\phi)$ dependency of the directivity from the frequency $(f)$ and distance $(r)$, the simulated directional wavefront will only be correct in the far-field. As with the present assumption regarding an omnidirectional velocity potential, the velocity components are in the radial direction of the simulated source only. Considering that the model is linear and time-invariant and that the near-field components may be modeled as an additive term for spherically symmetric wave propagation as discussed in Section III and given in (12), the fact that the model is not excited with the exact velocity values with both the near and far-field components does not impair the ability of the proposed method to simulate directivity of analytic sources in the far-field.

\section{B. Empirical Source Directivity}

1) Weighting in the Spatial Frequency Domain: The following procedure also summarized in Fig. 1 is carried out to simulate sound sources for which directivity functions measured in the acoustical far-field are available.

The measurements of the directivity function are assumed to be made at the measurement distance $R_{0}$ in the acoustical far-field of the source. Therefore, the directivity of the source is not known for $R<R_{0}$. In other words, the near-field effects are either neglected or assumed to be unknown. Let us also assume that the wavefront emitted by the source is spherical. This assumption allows using a monopole (i.e., an omnidirectional pressure source) to excite the DWM. The mesh can thus be initialized by setting the junction pressures and the simulation is started at the scattering step. The model is iterated for $n_{t}$ steps until the wavefront reaches the radius $R_{0}$ such that

$$
n_{t}=\left\lfloor\frac{R_{0} \sqrt{3}}{\Delta d}\right\rfloor=\left\lfloor\frac{R_{0} f_{S}}{c^{\prime}}\right\rfloor
$$

where $\lfloor\bullet\rfloor$ denotes the floor function. The outgoing wave variables $p_{i}^{-}\left(\hat{\mathbf{x}}, n_{t}\right)$ are obtained for all of the junctions in the model.

Empirical directivity functions are generally tabulated in octave-band resolution. This necessitates the decomposition of the numerical results obtained from the model into octave-bands before any weighting is carried out. This requires windowing in spatial frequency domain. Therefore, the obtained numerical results need to be transformed into spatial frequency domain first. Six $M \times M \times M$ element 3-D matrices of outgoing wave variables are obtained within a cubic volume $\mathcal{V}_{M} \cdot \mathcal{V}_{M}$ is selected in such a way that the center of the cubic volume is coincident with the point of excitation and the boundaries of the volume lie 


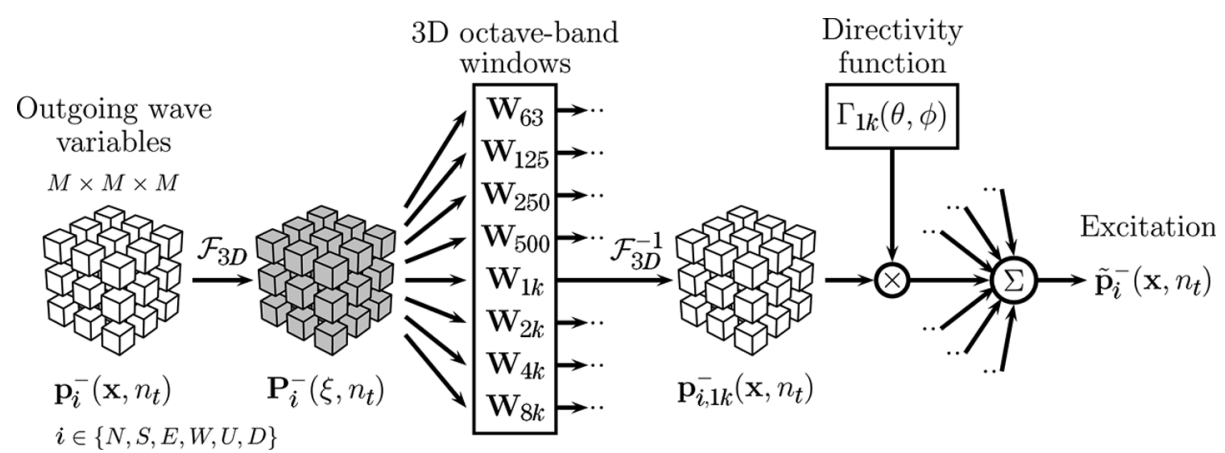

Fig. 1. The calculation of the directional outgoing wave variables $\tilde{\mathbf{p}}_{i}^{-}\left(\hat{\mathbf{x}}, n_{t}\right)$, where $\mathbf{p}_{i}^{-}\left(\hat{\mathbf{x}}, n_{t}\right)$ represents 3-D matrices of outgoing wave variables, $\mathbf{P}_{i}^{-}\left(\hat{\mathbf{x}}, n_{t}\right)$ represents the same matrix after 3-D discrete space Fourier transform is applied, $\mathbf{W}_{C}$ represents the 3-D octave-band window, and $\Gamma_{C}(\theta, \phi)$ represents the directivity function for the octave-band with a center frequency of $f_{C}$

within the undisturbed section of the DWM model. Let us denote these matrices and their representation in spatial frequency domain after applying the 3-D discrete space Fourier transform as

$$
\mathbf{p}_{i}^{-}\left(\hat{\mathbf{x}}, n_{t}\right) \stackrel{\mathcal{F}_{3 D}}{\longrightarrow} \mathbf{P}_{i}^{-}\left(\xi, n_{t}\right)
$$

where $i \in\{N, S, E, W, U, D\}$. Here, 3-D discrete-space Fourier transform is defined as

$$
\mathbf{P}_{i}^{-}(\xi, n)=\sum_{k=0}^{M-1} \sum_{l=0}^{M-1} \sum_{m=0}^{M-1} \mathbf{p}_{i}^{-}(\hat{\mathbf{x}}, n) e^{-j 2 \pi \xi \cdot \mathbf{x}}
$$

where $\mathbf{x}=[k \Delta d l \Delta d m \Delta d] \in \mathcal{V}_{M}$ and $\xi=\left[\xi_{x} \xi_{y} \xi_{z}\right]$ are the position and normalized spatial frequency vectors, respectively. If the 3-D discrete space Fourier transform is carried out using an $M$-point 3-D FFT, an $M \times M \times M$ 3-D matrix would be obtained.

To simulate a source with such directivity tabulated in octave-band resolution, components of the wavefront at those octave-bands need to be calculated. A windowing operation in the spatial frequency domain, which corresponds to zero-phase filtering, is carried out for calculating these octave-band components. The 3-D octave-band windows used in this operation are obtained in the following way.

The spatial frequencies $\xi=\left[\xi_{x} \xi_{y} \xi_{z}\right]$ are related to the temporal frequency $f_{t}$ in a 3-D DWM model as [46]

$$
f_{t}=\frac{f_{S}|\xi|}{\sqrt{3}}=f_{S} \sqrt{\left(\xi_{x}^{2}+\xi_{y}^{2}+\xi_{z}^{2}\right) / 3} .
$$

In other words, the normalized spatial frequencies and the temporal frequency can be used interchangeably in the context of DWM modeling as each discrete point in the spatial frequency domain corresponds to a single temporal frequency. For obtaining a 3-D octave-band window with a given temporal center frequency $f_{C}$, a prototype filter, $H_{C}(f)$ with the specifications given in IEC 61260:1995 [47] is designed as a sixth-order Butterworth filter. Using the correspondence between the temporal and spatial frequencies, the $M \times M \times M$ 3-D octave-band window, $\mathbf{W}_{C}(\xi)$, is obtained as

$$
\mathbf{W}_{C}(\xi)=\left|H_{C}\left(\frac{f_{S}|\xi|}{\sqrt{3}}\right)\right| \text {. }
$$

Note that this simple, real-valued 3-D window is spherically symmetric. The outgoing wave variable components at each octave-band are calculated in spatial frequency domain as

$$
\mathbf{P}_{i, C}^{-}\left(\xi, n_{t}\right)=\mathbf{P}_{i}^{-}\left(\xi, n_{t}\right) \bullet \mathbf{W}_{C}(\xi)
$$

where $\bullet$ denotes the Hadamard (i.e., elementwise) product of two matrices. The space domain components decomposed into octave-bands $\mathbf{p}_{i, C}^{-}\left(\hat{\mathbf{x}}, n_{t}\right)$ are obtained by applying an $M$-point 3-D IFFT. It should be noted that the output of the 3-D IFFT is a complex matrix for which the imaginary parts occurring due to the zero-phase octave-band windowing are negligibly small. Ideally, minimum-phase 3-D filters may be used to obtain the octave-band components directly in the space domain. However, spherical symmetry of the employed 3-D octave-band windows is a property which is hard if not impossible to obtain for 3-D filters designed using existing multivariate filter design techniques [48].

The outgoing wave variable components at each octave-band are then weighted in the space-domain with the directivity values at the corresponding octave-band in order to obtain the directional outgoing wave variables such that

$$
\tilde{\mathbf{p}}_{i, C}^{-}\left(\hat{\mathbf{x}}, n_{t}\right)=\Gamma_{C}(\theta, \phi) \mathbf{p}_{i, C}^{-}\left(\hat{\mathbf{x}}, n_{t}\right)
$$

where $\tilde{\mathbf{p}}_{i, C}^{-}$represents the directional outgoing wave variable components at the octave-band with center frequency $f_{C}$, and $\Gamma_{C}(\theta, \phi)$ is the value of the empirical directivity function at the same octave-band for the direction of the given point $\hat{\mathbf{x}}$ with respect to the origin of excitation.

The directional outgoing wave variables $\tilde{\mathbf{p}}_{i}^{-}\left(\hat{\mathbf{x}}, n_{t}\right)$ to be used for exciting the DWM model are obtained by the summation of the calculated outgoing wave variables $\tilde{\mathbf{p}}_{i, C}^{-}\left(\hat{\mathbf{x}}, n_{t}\right)$ for all the octave bands. The model is excited at the propagation step after setting the $\mathbf{p}_{i}^{-}$values accordingly. Starting with the iteration $n_{t}+1$, the wavefront will exhibit the directional characteristics of the simulated sound source. Algorithm 2 is a summary of the procedure described in this section. 
Algorithm 2 Empirical directivity

Initialize ROOM $\{\mathrm{ROOM}$ is the 3-D DWM model $\}$.

Excite ROOM with an omnidirectional pulse centered at $\bar{\mu}=\left[\mu_{x}, \mu_{y}, \mu_{z}\right]$.

for $n=1$ to $n_{t}$ do

propagate(ROOM)

scatter(ROOM)

end for.

Select an $M \times M \times M$ cubic volume $\mathcal{V}_{M}$ centered at $\bar{\mu}$.

Obtain the outgoing wave variables $\mathbf{p}_{k}^{-}\left(\hat{\mathbf{x}}, n_{t}\right)$ such that $k \in\{N, S, E, W, U, D\}$

for all $k$ such that $k \in\{N, S, E, W, U, D\}$ do

$\mathbf{P}_{k}^{-}\left(\xi, n_{t}\right)=\operatorname{FFT} 3\left[\mathbf{p}_{k}^{-}\left(\hat{\mathbf{x}}, n_{t}\right)\right]\{$ FFT3 is the $3-\mathrm{D}$ fast

Fourier transform $\}$

for all $f_{C} \in\{63,125,250,500,1 k, 2 k, 4 k, 8 k\}$ do

$\mathbf{P}_{k, C}^{-}\left(\xi, n_{t}\right)=\mathbf{W}_{C} \bullet \mathbf{P}_{k}^{-}\left(\xi, n_{t}\right)$

$\left\{\mathbf{W}_{C}\right.$ is the 3-D octave-band window at $\left.f_{C}\right\}$

$\mathbf{p}_{k, C}^{-}\left(\hat{\mathbf{x}}, n_{t}\right)=\operatorname{IFFT} 3\left[\mathbf{P}_{k, C}^{-}\left(\xi, n_{t}\right)\right]\{\operatorname{IFFT} 3$ is the 3-D inverse fast Fourier transform

for all $\mathbf{x}$ in $\mathcal{V}_{M}$ do

Calculate $\theta$ and $\phi$

$\tilde{\mathbf{p}}_{k, C}^{-}\left(\hat{\mathbf{x}}, n_{t}\right)=\Gamma_{C}(\theta, \phi) \mathbf{p}_{k, C}^{-}\left(\hat{\mathbf{x}}, n_{t}\right)\left\{\Gamma_{C}\right.$ is the directivity function at $\left.f_{C}\right\}$

end for

\section{end for}

$\tilde{\mathbf{p}}_{k}^{-}\left(\hat{\mathbf{x}}, n_{t}\right)=\sum_{C} \tilde{\mathbf{p}}_{k, C}^{-}\left(\hat{\mathbf{x}}, n_{t}\right)\{$ Sum of all weighted $3-\mathrm{D}$ octave-band matrices

end for.

Update the corresponding outgoing wave variables of ROOM with $\tilde{\mathbf{p}}_{k}^{-}\left(\hat{\mathbf{x}}, n_{t}\right)$

repeat

propagate $(\mathrm{ROOM})$

scatter(ROOM)

until the desired iteration count is reached.

Due to the linearity of the model, it is also possible to excite the mesh several times with different octave-band filtered pulses and obtain the simulation of the corresponding directional wavefront by the summation of the results of these simulations.

The method detailed above assumes the knowledge of the directivity function for all possible directions. However, directivity measurements of real sources are generally tabulated not for every possible direction around the source, but only for the horizontal and vertical planes making it problematic to apply the available measurements directly. Therefore, it is necessary to obtain a closed-form representation for the directivity function from the incomplete directivity measurements. A simple functional interpolation method based on curve fitting is described in the following section.

2) Interpolation of Directivity Functions: Let us assume that two sets of directivity measurements, $\Gamma_{C, h}(\theta, 0)$ and $\Gamma_{C, v}(0, \phi)$, are available on the horizontal and vertical planes of the sound source, respectively, at the octave bands with center frequencies $f_{C}$. The interpolation of a directivity function is the process of obtaining a directivity function defined for all possible directions from a set of incomplete measurements made at a limited set of directions. In other words, the aim is to obtain a closed-form function $\Gamma_{C}^{\prime}(\theta, \phi)$ for each $f_{C}$ that approximates the underlying (i.e., actual) directivity function from which a limited set of measurements is available. The process of obtaining an analytical expression from the measured data is also known as curve-fitting compaction [49] as it allows the compact storage of the underlying, measured directivity data.

The data from measurements on the horizontal and vertical planes at a given octave band are first fit to closed curves using shape-preserving piecewise cubic splines such that

$$
\begin{aligned}
& \Gamma_{C}(\theta) \approx \Gamma_{C, h}^{\prime}(\theta, 0)=Q_{C, h}(\theta) \\
& \Gamma_{C}(\phi) \approx \Gamma_{C, v}^{\prime}(0, \phi)=Q_{C, v}(\phi)
\end{aligned}
$$

where $Q_{C, h}$ and $Q_{C, v}$ are piecewise functions approximating the shape of the original measurement points on horizontal and vertical planes, respectively. In order to obtain the values of the directivity function for any given direction, the following interpolation is carried out:

$$
\Gamma_{C}^{\prime}(\theta, \phi)=Q_{C, h}(\theta) \rho(\theta, \phi)+Q_{C, v}(\phi)[1-\rho(\theta, \phi)]
$$

where $\rho(\theta, \phi)$ is a smooth function and $0 \leq \rho(\theta, \phi) \leq 1$. Ideally, $\rho(m \pi, \phi)=0$ and $\rho(\theta, n \pi)=1$ so that the interpolated function is exactly equal to $Q_{C, h}(\theta)$ on the horizontal plane, and to $Q_{C, v}(\phi)$ on the vertical plane. However, such a smooth bivariate function does not exist due to discontinuities occurring at $\theta= \pm m \pi$, and $\phi= \pm n \pi$. Therefore an approximate function is selected such that

$$
\rho(\theta, \phi)=0.5\left[\sin ^{2} \theta+\cos ^{2} \phi\right] .
$$

The closed-form definition of the directivity function allows the interpolation of intermediate values between horizontal and vertical planes. In addition, it allows easy rotation of the modeled directivity function in azimuth and elevation.

Fig. 2 shows the interpolated directivity function for a baritone singer obtained from [50]. The interpolated directivities on horizontal; i.e., $Q_{1 k, h}(\theta)$, and vertical; i.e., $Q_{1 k, v}(\phi)$, planes and the interpolated directivity balloon for $f_{C}=1 \mathrm{kHz}$ are shown. Although the applied interpolation is not based on a physical interpretation, it is preferred for its simplicity and generality. 


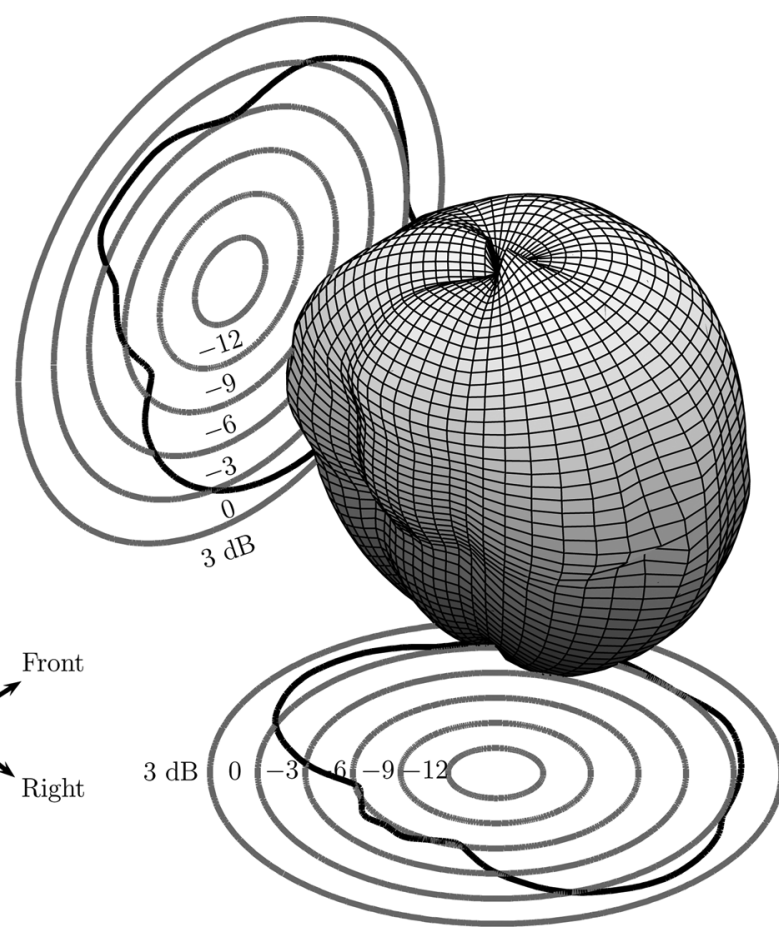

Fig. 2. Interpolated directivity of a baritone singer for the octave band with the center frequency $f_{C}=1 \mathrm{kHz}$. The projections to the left and bottom planes show the curves fit to data for vertical and horizontal measurements, respectively. The volume shows the interpolated directivity balloon.

\section{Simulations on a 3-D Digital WaVeguide Mesh}

\section{A. Employed 3-D DWM Model}

The DWM used in the simulations was a uniformly sampled rectilinear mesh with $458 \times 352 \times 302$ elements modeling a rectangular room. At the temporal sampling frequency of $f_{S}=$ $44.1 \mathrm{kHz}$ and for the speed of sound $c=344 \mathrm{~m} / \mathrm{s}$, the interjunction distance $\Delta d \approx 0.0135 \mathrm{~m}$, and the physical size of the modeled enclosure is approximately $6.19 \mathrm{~m} \times 4.76 \mathrm{~m} \times 4.08 \mathrm{~m}$. The boundaries were selected to be fully reflective at all frequency bands, i.e., the absorption coefficient $\alpha=0$.

As the proposed methods need to access the outgoing wave variables, the memory requirements of the DWM model needed to be doubled. The resulting memory requirements of the mesh made it necessary to employ a single precision implementation instead of double precision. By employing such design considerations, the size of the 3-D DWM model was approximately 2.2 GB. For speeding up the simulation, Altivec extensions of the PowerPC architecture were employed and the code was hand-optimized. The system over which the simulation was executed had 4 PPC 970MP CPU cores with 1 MB L2 cache each, and an system memory of $8 \mathrm{GBs}$. Two directional sources were simulated. The first source was an ideal dipole with an analytical directivity. The second was a baritone singer with empirical directivity data tabulated in octave-band resolution.

In the following sections, the positions of the junctions in the model at which the simulated directivities were obtained do not exactly correspond to the actual intended directions and measurement distance due to discretization. However, the direction

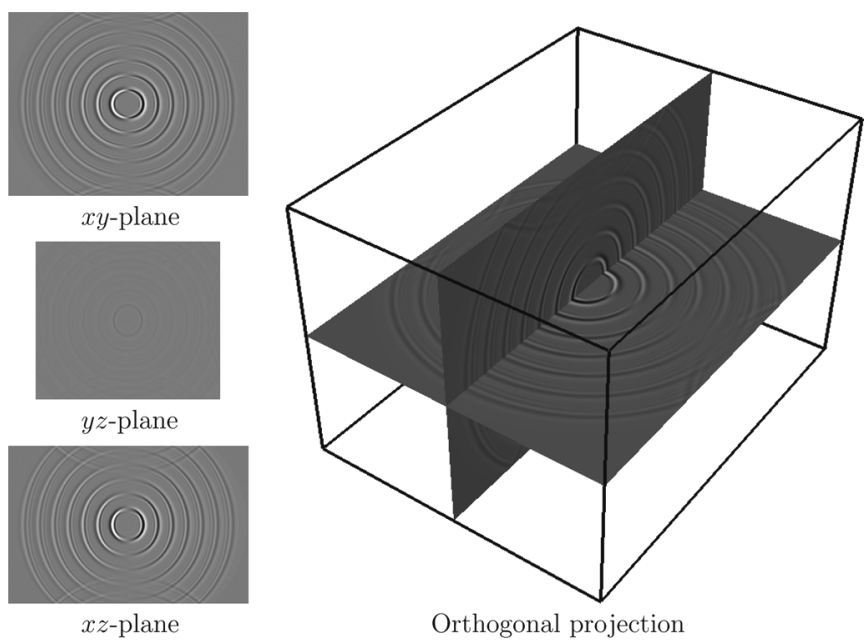

Fig. 3. Visualization of the pressure field on the 3-D DWM model excited with an ideal dipole source showing the superimposed wavefronts at the iterations $n_{t}=50,100,150,200,250,300$, and 350. The left column shows the propagation in $x y, y z$, and $x z$ planes. The right column shows the orthogonal projection of the $x y$ and $x z$ planes. Colormap ranges from black (minimum) to white (maximum).

and distance uncertainty may be considered to be negligible at the distance at which the directivity was obtained.

\section{B. Analytical Directivity: Ideal Dipole}

The ideal dipole is an approximation of a point velocity source propagating two opposite phase wavefronts toward opposite directions. A simple mathematical expression exists for the far-field directivity of the ideal dipole such as

$$
\Gamma(\theta, \phi)=\cos \theta \cos \phi .
$$

The wave propagation is stronger in the front-back axis of the dipole. There is no wave propagation on the plane perpendicular to the look direction which also constitutes the nulls of the directivity function (i.e., $\theta= \pm \pi / 2$ and $\phi= \pm \pi / 2$ ).

For simulating an ideal dipole, a trivariate Gaussian velocity potential with a standard deviation of 0.04 was selected as described in (19). It was normalized to have values between 0 and 1 , and sampled on a $102 \times 102 \times 102$ uniform rectilinear grid. The initial velocity field to be used for exciting the DWM was calculated by obtaining the numerical gradient of the sampled velocity potential, and weighting the obtained omnidirectional velocity field by the analytical directivity function.

Fig. 3 shows the superimposed sound fields for the iteration steps $n=50,100,150,200,250,300$, and 350 at the $x y, y z$, and $x z$ planes and an orthogonal projection as visualized using the volumetric visualization program, ParaView [51]. It may be observed that there is no wave propagation in the $y z$-plane that coincides with the nulls of the directivity function, while the wavefronts in $+x$ and $-x$ directions have opposite phases.

Fig. 4 shows the directivity function for horizontal and vertical planes obtained on the mesh at a distance $R_{0}=1 \mathrm{~m}$ corresponding to a discrete sphere of radius $R_{\Delta}=74 \Delta d$ for the $f_{C}=2 \mathrm{kHz}$ band. The ideal directivity function was also plotted for comparison. The angular interval between the sampling points on both the horizontal and the vertical planes was 


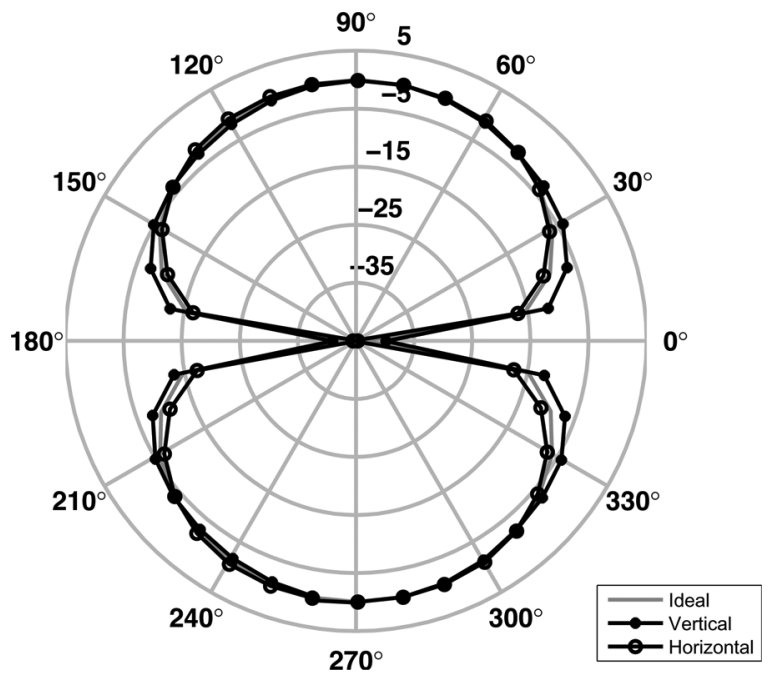

Fig. 4. Simulated and ideal dipole directivity functions at the octave-band with the center frequency $f_{C}=2 \mathrm{kHz}$. Open and filled circles represent simulated directivity sampled on the horizontal and vertical planes of the simulated dipole source, respectively. The gray solid line is the ideal dipole directivity function.

TABLE I

MAXIMUM $\left(\epsilon_{\max }\right)$ AND AVERAGE $\left(\epsilon_{\text {avg }}\right)$ ABSOLUTE APPROXIMATION ERRORS IN dB BETWEEN THE INTENDED AND SiMUlated IdEAL DiPOLE DiRECTIVITY ON HORIZONTAL $\left(\phi=0^{\circ}\right)$ AND VERTICAL $\left(\theta=0^{\circ}\right)$ PLANES

\begin{tabular}{|c|c|c|c|c|c|c|c|}
\hline & & \multicolumn{6}{|c|}{$f_{C}(\mathrm{~Hz})$} \\
\hline & & 125 & 250 & 500 & 1000 & 2000 & 4000 \\
\hline \multirow{2}{*}{$\begin{array}{c}\text { for } \\
\phi=0\end{array}$} & $\epsilon_{\max }$ & 0.57 & 0.49 & 0.49 & 0.58 & 2.19 & 5.67 \\
\hline & $\epsilon_{\text {avg }}$ & 0.18 & 0.14 & 0.12 & 0.13 & 0.58 & 1.90 \\
\hline \multirow{2}{*}{$\begin{array}{c}\text { for } \\
\theta=0\end{array}$} & $\epsilon_{\max }$ & 1.16 & 1.17 & 1.94 & 3.16 & 3.73 & 4.98 \\
\hline & $\epsilon_{\text {avg }}$ & 0.46 & 0.38 & 0.48 & 0.96 & 0.97 & 1.99 \\
\hline
\end{tabular}

chosen as $10^{\circ}$ for illustration purposes. As may be observed, the sampled directivity on the mesh have a good correspondence with the intended ideal dipole characteristics.

Table I summarizes the maximum $\left(\epsilon_{\max }\right)$ and average $\left(\epsilon_{\text {avg }}\right)$ absolute errors between the ideal and the simulated dipole directivity. We speculate without further elaboration that the errors involved in the simulation of the analytical directivity are due to the direction dependent dispersion. Direction dependence of the dispersion error may be reduced by using other mesh topologies, or by using interpolated meshes [52]. In addition, existing techniques such as frequency warping [46] may be used with interpolated meshes to reduce the dispersion error.

\section{Empirical Directivity: Baritone Singer}

Directivity measurements of a baritone singer were obtained from Marshall and Meyer [50], and interpolation was carried out as defined above. The mesh was excited with an omnidirectional pressure field and iterated for $n_{t}=128$ steps corresponding to $R_{0} \simeq 1 \mathrm{~m}$. The original excitation was a normalized trivariate Gaussian function with a standard deviation of 0.04 . The outgoing wave variables were obtained in a $302 \times 302 \times 302$-element cubic volume with a center coincident with the original point of excitation. The values corresponding to the velocity components were transformed into spatial frequency domain via a 302-point 3-D FFT and windowed with 3-D octave-band windows at $f_{C}=125,250,500,1000,2000,4000 \mathrm{~Hz}$. The

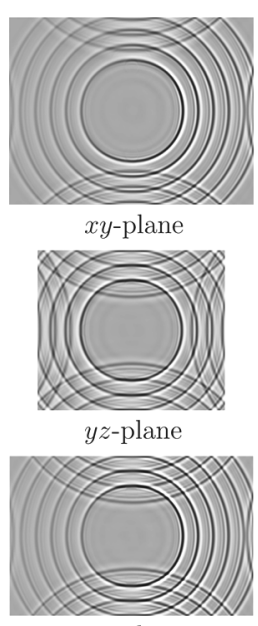

$x z$-plane

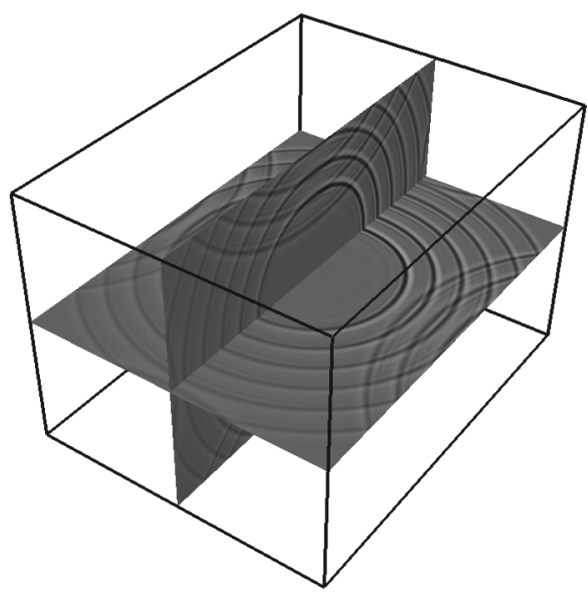

Orthogonal projection
Fig. 5. Visualization of the pressure field on the 3-D DWM model excited with a directional source simulating a baritone singer. The superimposed wavefronts at the iterations $n_{t}=150,200,250,300,350$, and 400 are shown. The left column shows the propagation in $x y, y z$, and $x z$ planes. The right column shows the orthogonal projection of the $x y$ and $x z$ planes. Colormap ranges from black (minimum) to white (maximum).

upper frequency limit for the simulation at the given temporal sampling frequency is $f_{u}=11025 \mathrm{~Hz}$. This value is lower than the higher cutoff frequency of the octave-band filter with $f_{C}=8 \mathrm{kHz}$. Therefore, the octave-band with $f_{C}=8 \mathrm{kHz}$ was not included in the simulation so as to prevent errors due to aliasing. The space domain octave-band components were obtained as the real portion of the results of a 3-D IFFT. Directional weighting was carried out at each octave-band and the new full bandwidth outgoing wave variables were obtained as a summation of these directional octave-band components. The obtained outgoing wave variables were used for exciting the model. The iterations were restarted at the propagation step.

It should be noted that although the re-excitation of the model with the wavefront at $1 \mathrm{~m}$ reduces the effect of the near-field component, it does not completely eliminate it. Some sound sources such as musical instruments have significant near-field effects at that distance, particularly at lower frequencies. However, as long as tabulated far-field directivity functions are considered, the lack of information on the full field variables, specifically the angular dependency of the particle velocity makes it impossible to obtain a more accurate simulation.

Fig. 5 shows the visualization of the superimposed sound fields for the iteration steps $n_{t}=150,200,250,300,350$, and 400 for $x y, y z$, and $x z$ planes and as an orthogonal projection. It may be observed that the wavefronts are strongly directional in the look direction of the simulated virtual source. The portion of the wavefront propagating toward the $-x$ direction is significantly attenuated in comparison with the $+x$ direction as would be expected from a singer facing away.

Figs. 6 and 7 show the simulated directivity sampled on the mesh for the horizontal and vertical planes at a distance $R=$ $1.5 \mathrm{~m}$ corresponding to a discrete sphere of radius $R_{\Delta} \approx 110 \Delta d$ for the octave-bands with center frequencies $f_{C}=125,250$, $500,1000,2000,4000 \mathrm{~Hz}$. The interpolated directivity functions 


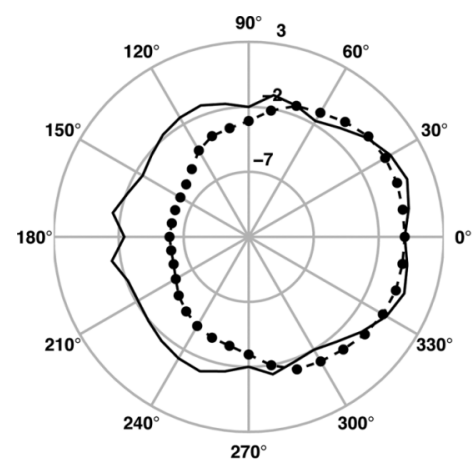

$f_{c}=125 \mathrm{~Hz}$

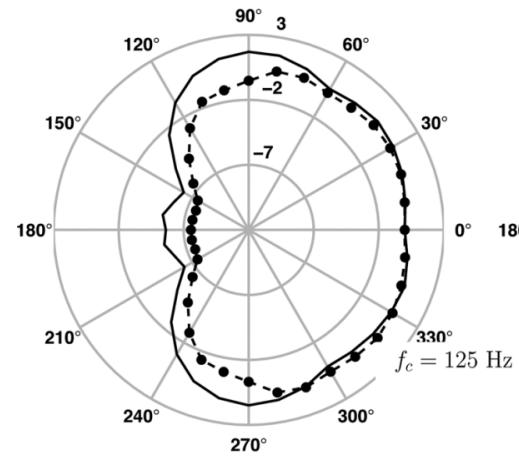

$f_{c}=1 \mathrm{kHz}$

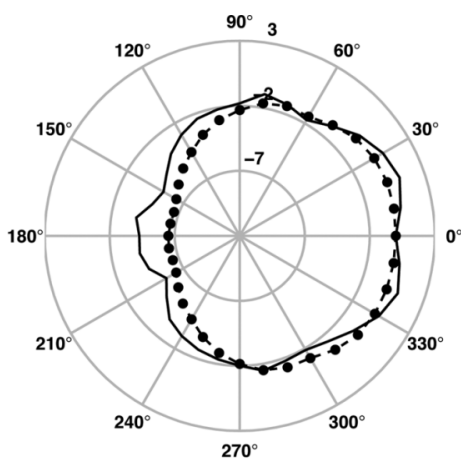

$f_{c}=250 \mathrm{~Hz}$

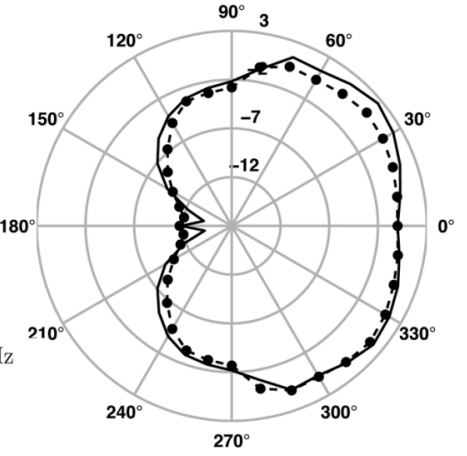

$f_{c}=2 \mathrm{kHz}$

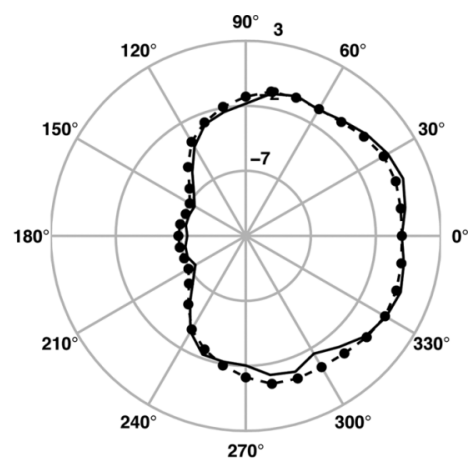

$f_{c}=500 \mathrm{~Hz}$

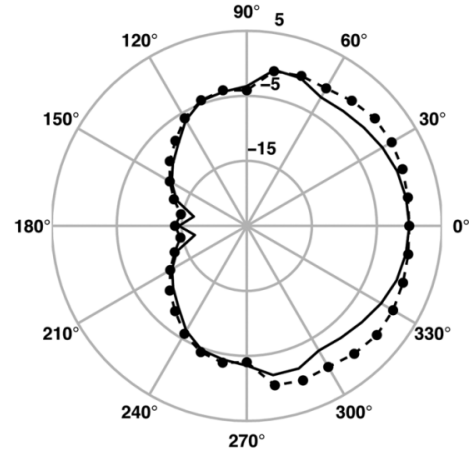

$f_{c}=4 \mathrm{kHz}$

Fig. 6. Simulated and ideal directivity functions $\Gamma_{C}(\theta, \phi)$ on the horizontal plane of the simulated baritone singer for the octave-bands with center frequencies $f_{C}=125,250,500,1000,2000,4000 \mathrm{~Hz}$. The solid lines represent the intended directivity functions. The filled points connected with broken lines represent the directivity function sampled on the 3-D DWM model. Note that the scale is not the same in all frequency bands.

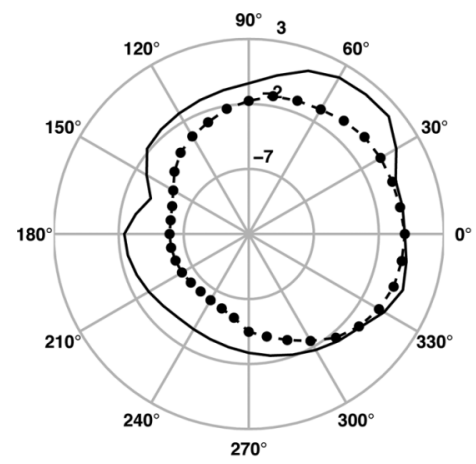

$f_{c}=125 \mathrm{~Hz}$

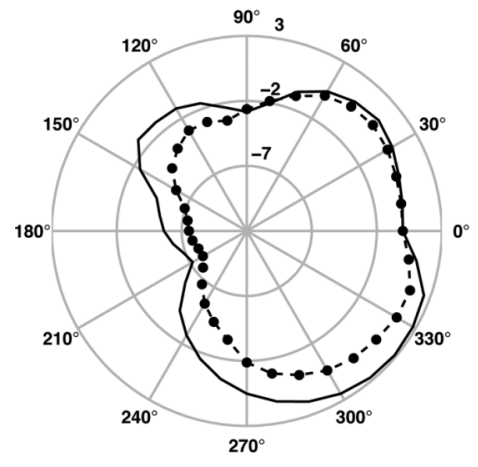

$f_{c}=1 \mathrm{kHz}$

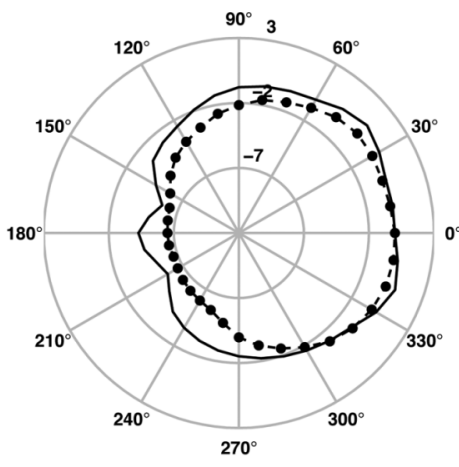

$f_{c}=250 \mathrm{~Hz}$

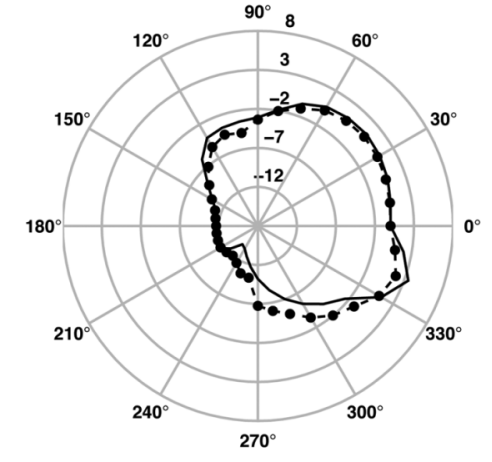

$f_{c}=2 \mathrm{kHz}$

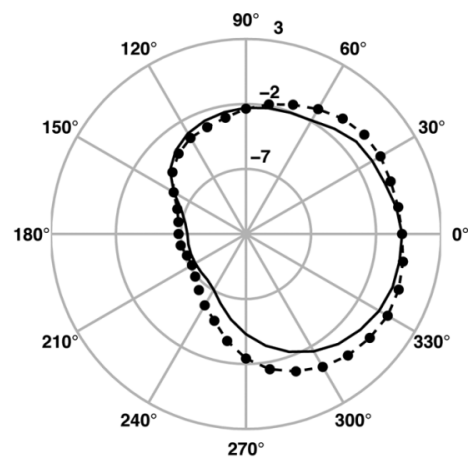

$f_{c}=500 \mathrm{~Hz}$

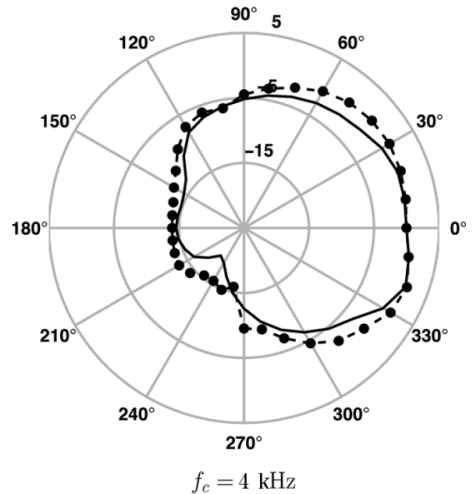

Fig. 7. Simulated and ideal directivity functions $\Gamma_{C}(\theta, \phi)$ on the vertical plane for the simulated baritone singer for the octave-bands with center frequencies $f_{C}=125,250,500,1000,2000,4000 \mathrm{~Hz}$. The solid lines represent the intended directivity functions. The filled points connected with broken lines represent the directivity function sampled on the 3-D DWM model. Note that the scale is not the same in all frequency bands.

in the respective frequency bands are also shown for comparison. As with the analytical directivity simulation example, the angular interval between the sampling points was chosen as $10^{\circ}$ for illustration purposes. Initially, the response of the mesh to 
TABLE II

$\operatorname{MAXIMUM}\left(\epsilon_{\max }\right)$ AND AVERAGE $\left(\epsilon_{\mathrm{avg}}\right)$ APPROXIMATION ERRORS IN dB BETWEEN THE INTENDED AND SIMULATED BARITONE DIRECTIVITY ON THE Horizontal $\left(\phi=0^{\circ}\right)$ AND Vertical $\left(\theta=0^{\circ}\right)$ Planes

\begin{tabular}{|c|c|c|c|c|c|c|c|}
\hline & \multicolumn{6}{|c|}{$f_{C}(\mathrm{~Hz})$} \\
\hline & & 125 & 250 & 500 & 1000 & 2000 & 4000 \\
\hline for & $\epsilon_{\max }$ & 4.63 & 2.67 & 1.19 & 2.46 & 2.21 & 3.21 \\
\hline$\phi=0$ & $\epsilon_{\mathrm{avg}}$ & 1.81 & 0.92 & 0.41 & 1.20 & 0.76 & 1.04 \\
\hline for & $\epsilon_{\max }$ & 3.47 & 2.52 & 1.86 & 3.41 & 3.45 & 3.98 \\
\hline$\theta=0$ & $\epsilon_{\text {avg }}$ & 1.81 & 1.12 & 0.76 & 1.57 & 1.03 & 1.57 \\
\hline
\end{tabular}
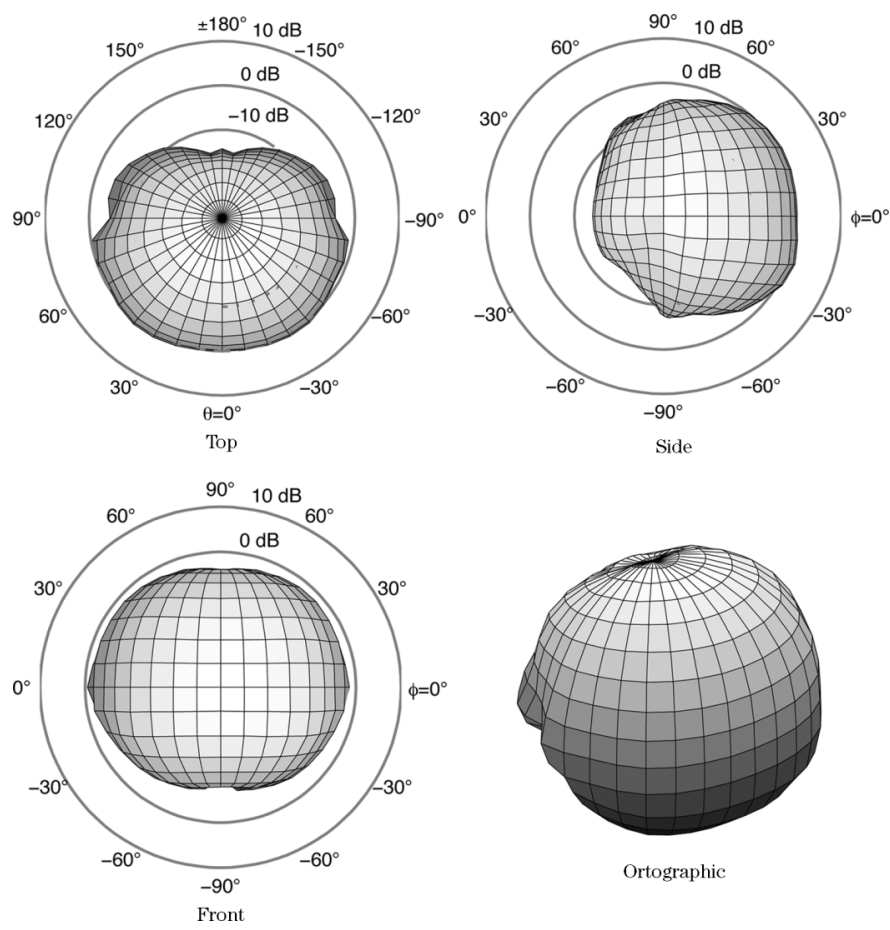

Fig. 8. Directivity balloon for the simulated baritone at the octave-band with the center frequency $f_{C}=4 \mathrm{kHz}$. The figure shows the front, top, side, and orthographic views.

the directional wavefront was obtained by registering the fluctuation of junction pressure values at the sampling points prior to the arrival of any reflection at those points. These sequences are then filtered into octave-bands by using sixth-order Butterworth octave-band filters. The directivity values were obtained by calculating the rms amplitudes of these octave-band components and normalizing the obtained values to correspond to $0 \mathrm{~dB}$ for the front direction. The results present a good correspondence between the original and the simulated directivities. Note that the ranges of the polar plots are different in some of the frequency bands.

Table II shows the maximum and average errors in $\mathrm{dB}$ for horizontal and vertical planes in each frequency band. It may be observed that errors are higher in general for the $f_{C}=125 \mathrm{~Hz}$ band. This may be due to the low spatial frequency resolution of the 3-D FFT for the given data size. It should be noted that normalization with respect to an axial direction of the cubic mesh also increases the maximum and average errors as direction dependent dispersion will be more prominent in axial directions specifically at higher frequency bands.
Fig. 8 shows the directivity at $f_{C}=4 \mathrm{kHz}$ obtained from the simulation on the DWM on 648 sample points around the simulated source with $10^{\circ}$ separation both in azimuth and elevation.

\section{CONCLUSION}

Two methods for simulating source directivity within 3-D DWM models were presented in this paper. The first method is used for simulating a point source with an analytical directivity function. The DWM is excited with directionally weighted outgoing wave variables, and the iteration is started at the propagation step. The simulated directive wavefront will have the directive properties of the source in the far-field. The second method is used for simulating a sound source with an empirical directivity function measured at the acoustical far-field. The DWM is excited with an omnidirectional point source and iterated to allow the resulting wavefront to arrive at the acoustical far-field. The resulting wavefront is weighted with the directivity function at different octave-bands. This method requires the knowledge of the complete directivity function at all points around the source. However, generally, only a limited set of measurements is tabulated in literature. To overcome this difficulty, a simple interpolation method to obtain a functional description of the directivity function was also described. Two application examples on a large uniformly sampled rectilinear 3-D DWM model of a room were given for the analytical and the empirical directivity cases. It was shown that the method can be successfully utilized for simulating directional sources. The simulation results for an ideal dipole show that the maximum absolute error between the simulated and original directivity values is observed at the $4-\mathrm{kHz}$ octave-band and is around $5 \mathrm{~dB}$. The average absolute error is less that $2 \mathrm{~dB}$ for all frequency bands. The simulation results for a source with an empirical directivity function show that the maximum absolute error is lower than $5 \mathrm{~dB}$ and the average error is less than $2 \mathrm{~dB}$ in all frequency bands. Although the method was presented for uniformly sampled rectilinear 3-D DWMs in this paper, it can easily be applied to other DWM topologies with slight modifications.

\section{ACKNOWLEDGMENT}

The authors would like to thank the Associate Editor Prof. V. Välimäki and the three anonymous reviewers for their feedback and insightful comments.

\section{REFERENCES}

[1] A. Krokstad, S. Strom, and S. Sørsdal, "Calculating the acoustical room response by the use of a ray tracing technique," J. Sound Vibr., vol. 8, no. 1 , pp. 118-125, Jun. 1968

[2] T. Funkhouser, N. Tsingos, I. Carlbrom, G. Elko, M. Sondhi, J. E. West, G. Pingali, P. Min, and A. Ngan, "A beam tracing method for interactive architectural acoustics," J. Acoust. Soc. Amer., vol. 115, no. 2, pp. 739-756, Feb. 2004.

[3] J. B. Allen and D. A. Berkley, "Image method for efficiently simulating small-room acoustics," J. Acoust. Soc. Amer., vol. 65, no. 4, pp. 943-950, Apr. 1979.

[4] P. Svensson and U. R. Kristiansen, "Computational modeling and simulation of acoustic spaces," in Proc. AES 22nd Int. Conf. Virtual, Synth., Entertainment Audio (AES22), Espoo, Finland, Jun. 15-17, 2002, pp. 11-30. 
[5] A. Craggs, "Acoutical modeling: Finite element method," in Handbook of Acoustics, M. J. Crocker, Ed. New York: Wiley, 1998, ch. 12, pp. $149-156$.

[6] A. F. Seybert and T. W. Wu, "Acoustic modeling: Boundary element methods," in Handbook of Acoustics, M. J. Crocker, Ed. New York: Wiley, 1998, ch. 13, pp. 157-167.

[7] D. Botteldooren, "Acoustical finite-difference time-domain simulation in a quasi-Cartesian grid," J. Acoust. Soc. Amer., vol. 95, no. 5, pp. 2313-2319, May 1994.

[8] D. Botteldooren, "Finite-difference time-domain simulation of lowfrequency room acoustic problems," J. Acoust. Soc. Amer., vol. 98, no. 6, pp. 3302-3308, Dec. 1995.

[9] T. Schetelig and R. Rabenstein, "Simulation of three-dimensional sound propagation with multidimensional wave digital filters," in Proc. 1998 IEEE Int. Conf. Acoust., Speech, Signal Process. (ICASSP'98), Seattle, WA, May 12-15, 1998, vol. 6, pp. 3537-3540.

[10] J. O. Smith, III, "Physical modeling using digital waveguides," Comput. Music J., vol. 16, no. 4, pp. 74-91, Winter, 1992.

[11] J. Borish, "Extension of the image model to arbitrary polyhedra," $J$. Acoust. Soc. Amer., vol. 75, no. 6, pp. 1827-1836, Jun. 1984.

[12] J. O. Smith, III, "On the equivalence of the digital waveguide and finite difference time domain schemes," [Online]. Available: http://arxiv.org/ abs/physics/0407032

[13] J. L. Flanagan, "Analog measurements of sound radiation from the mouth," J. Acoust. Soc. Amer., vol. 32, no. 12, pp. 1613-1620, Dec. 1960.

[14] J. Huopaniemi, K. Kettunen, and J. Rahkonen, "Measurement and modelling techniques for directional sound radiation from the mouth," in Proc. IEEE Workshop Appl. Signal Process. Audio Acoust. (WASPAA'99), New Paltz, NY, Oct. 17-20, 1999, pp. 183-186.

[15] T. Halkosaari, M. Vaalgamaa, and M. Karjalainen, "Directivity of artificial and human speech," J. Audio Eng. Soc., vol. 53, no. 7/8, pp. 620-631, Jul./Aug. 2005.

[16] J. Meyer, Acoustics and the Performance of Music. Frankfurt/Main, Germany: Verlag Das Musikinstrument, 1978.

[17] A. Farina, "Pyramid tracing vs. ray tracing for the simulation of sound propagation in large rooms," in Computational Acoustics and Its Environmental Applications, C. Brebbia, Ed. Southampton, U.K.: Computational Mechanics Publications, 1995, pp. 109-116.

[18] J. Huopaniemi, "Virtual acoustics and 3-D sound in multimedia signal processing," Ph.D. dissertation, Dept. Elect. Commun. Eng., Helsinki Univ. of Technol., Espoo, Finland, 1999.

[19] L. Cremer and M. Wang, "Synthesis of spherical wave fields to generate the sound radiated from bodies of arbitrary shape, its realisation by calculation and experiment," (in German) Acustica, vol. 65, pp. 53-74, 1988.

[20] F. Giron, "Investigations About the Directivity of Sound Sources," $\mathrm{Ph} . D$. dissertation, Institut für Kommunikationsakustik, Ruhr-Universität Bochum, , Nov. 1996.

[21] R. Jeans and C. Mathews, "The wave superposition method as a robust technique for computing acoustic fields," J. Acoust. Soc. Amer., vol. 92, no. 2, pp. 1156-1166, Aug. 1992.

[22] G. H. Koopmann, L. Song, and J. B. Fahnline, "A method for computing acoustic fields based on the principle of wave superposition," $J$. Acoust. Soc. Amer., vol. 86, no. 6, pp. 2433-2438, Dec. 1989.

[23] M. Ochmann, "The source simulation technique for acoustic radiation problems," Acustica, vol. 81, no. 6, pp. 512-527, Nov. 1995.

[24] W. Kropp and P. U. Svensson, "Application of the time domain formulation of the method of equivalent sources to radiation and scattering problems," Acustica, vol. 81, no. 6, pp. 528-543, Nov. 1995.

[25] H. Hacıhabiboğlu, B. Günel, and A. M. Kondoz, "Source directivity simulation in digital waveguide mesh-based room acoustics models," in Proc. AES 30th Int. Conf. Intell. Audio Environments, Mar. 15-17, 2007, CD-ROM.

[26] J. Escolano, J. J. López, and B. Pueo, "Directive sources in acoustic discrete-time domain simulations based on directivity diagrams," $J$. Acoust. Soc. Amer., vol. 121, no. 6, pp. EL256-EL262, Jun. 2007.

[27] C. Erkut, "Aspects in analysis and model-based sound synthesis of plucked string instruments," Ph.D. dissertation, Dept. Elect. Commun. Eng., Helsinki Univ. of Technol., Espoo, Finland, Nov. 2002.

[28] J. Mullen, D. M. Howard, and D. T. Murphy, "Real-time dynamic articulations in the 2-D waveguide mesh vocal tract model," IEEE Trans. Audio, Speech. Lang. Process., vol. 15, no. 2, pp. 577-585, Feb. 2007.
[29] J. Laird, P. Masri, and C. N. Canagarajah, "Efficient and accurate synthesis of circular membranes using digital waveguides," in Proc. Inst. Elect. Eng. Colloquium Audio and Music Technol.: The Challenge of Creative DSP, Nov. 18, 1998, pp. 12/1-12/6.

[30] L. Savioja, T. Rinne, and T. Takala, "Simulation of room acoustics with a 3-D finite difference mesh," in Proc. Int. Comput. Music Conf. (ICMC'94), Aarhus, Denmark, Sep. 1994, pp. 463-466.

[31] D. T. Murphy and D. M. Howard, "Modelling and directionally encoding the acoustics of a room," Electron. Lett., vol. 34, no. 9, pp. 864-865, Apr. 1998.

[32] A. Kelloniemi, V. Välimäki, and L. Savioja, "Simulation of room acoustics using 2-D digital waveguide meshes," in Proc. 2006 IEEE Int. Conf. Acoust., Speech, Signal Process. (ICASSP'06), May 15-19, 2006, vol. 5, pp. 313-316.

[33] M. Karjalainen and C. Erkut, "Digital waveguides versus finite-difference structures: Equivalence and mixed modeling," EURASIP J. Appl. Signal Process., vol. 2004, no. 7, pp. 978-989, 2004.

[34] D. T. Murphy and M. Beeson, "The KW-boundary hybrid digital waveguide mesh for room acoustics applications," IEEE Trans. Audio, Speech, Lang. Process., vol. 15, no. 2, pp. 552-564, Feb. 2007.

[35] S. A. Van Duyne and J. O. Smith, III, "The 2-D digital waveguide mesh," in Proc. 1993 IEEE Workshop Appl. Signal Process. Audio and Acoust. (WASPAA'93), New Paltz, NY, Oct. 17-20, 1993, pp. 177-180.

[36] S. Bilbao, Wave and Scattering Methods for Numerical Simulation. Chichester, U.K.: Wiley, 2004.

[37] S. A. Van Duyne and J. O. Smith, III, "The tetrahedral digital waveguide mesh," in Proc. IEEE Workshop Appl. Signal Process. Audio and Acoust. (WASPAA'95), New Paltz, NY, Oct. 15-18, 1995, pp. 234-237.

[38] G. R. Campos and D. M. Howard, "On the computational efficiency of different waveguide mesh topologies for room acoustics simulation," IEEE Trans. Speech Audio Process., vol. 13, no. 5, pp. 1063-1072, Sep. 2005.

[39] H. Hacıhabiboğlu, B. Günel, and A. M. Kondoz, "On the accuracy of first-order numerical derivatives in multidimensional digital waveguide mesh topologies," IEEE Signal Process. Lett., vol. 15, no. 1, pp. 9-12, Jan. 2008.

[40] A. Villareal and J. A. Scales, "Distributed three-dimensional finite-difference modelling of wave propagation in acoustic media," Comput. Phys., vol. 11, no. 4, pp. 388-399, Jul./Aug. 1997.

[41] G. Campos and D. M. Howard, "On the computation time of threedimensional digital waveguide mesh acoustic models," in Proc. 26th Euromicro Conf., Maastricht, The Netherlands, May 5-7, 2000, pp. 332-339.

[42] S. Bilbao and J. O. Smith, III, "Finite difference schemes and digital waveguide networks for the wave equation: Stability, passivity, and numerical dispersion," IEEE Trans. Speech Audio Process., vol. 11, no. 3, pp. 255-266, May 2003.

[43] A. D. Pierce, Acoustics: An Introduction to Its Physical Principles and Applications. Woodbury, NY: Acoust. Soc. Amer., 1994.

[44] J. Escolano and F. Jacobsen, "A note on the physical interpretation of frequency dependent boundary conditions in a digital waveguide mesh," Acta Acustica United With Acustica, vol. 93, no. 3, pp. 398-402, May/Jun. 2007.

[45] H. Kuttruff, Room Acoustics. $\quad$ London, U.K.: Applied Science, 1979.

[46] L. Savioja and V. Välimäki, "Reducing the dispersion error in the digital waveguide mesh using interpolation and frequency-warping techniques," IEEE Trans. Speech Audio Process., vol. 8, no. 2, pp. 184-194, Mar. 2000.

[47] Electroacoustics-Octave-Band and Fractional-Octave-Band Filters, IEC 61260:1996, Int. 1 Electrotech. Commission, 1996.

[48] M. E. Zervakis and A. N. Venetsanopoulos, "Design of three-dimensional digital filters using two-dimensional rotated filters," IEEE Trans. Circuits Syst., vol. 34, no. 12, pp. 1452-1469, Dec. 1987.

[49] M. H. Weik, Communications Standard Dictionary, 3rd ed. New York: Springer, 1996

[50] A. H. Marshall and J. Meyer, "The directivity and auditory impressions of singers," Acustica, vol. 58, no. 3, pp. 130-140, 1985

[51] J. Ahrens, B. Geveci, and C. Law, "ParaView: An end-user tool for large data visualization," in Visualization Handbook, C. D. Hansen and C. R. Johnson, Eds. New York: Elsevier, 2005, ch. 36, pp. 717-732.

[52] L. Savioja and V. Välimäki, "Interpolated rectangular 3-D digital waveguide mesh algorithms with frequency warping," IEEE Trans. Speech Audio Process., vol. 11, no. 6, pp. 783-790, Nov. 2003. 


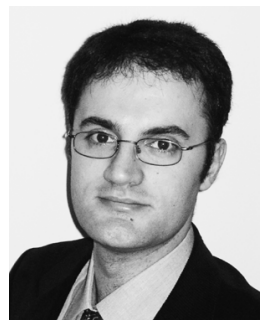

Hüseyin Hacıhabiboğlu (S'96-M'00) was born in Ankara, Turkey, in 1978. He received the B.Sc. (Hons.) degree from the Middle East Technical University, Ankara, in 2000, the M.Sc. degree from the University of Bristol, Bristol, U.K., in 2001, both in electrical and electronic engineering, and the $\mathrm{Ph} . \mathrm{D}$. degree in computer science from Queens University Belfast, Belfast, U.K., in 2004.

From 2004 to 2008, he was with the Multimedia and DSP Research Group (I-Lab), Centre for Communication Systems Research (CCSR), University of Surrey, Guildford, U.K. He is currently with the Centre for Digital Signal Processing Research (CDSPR), Division of Engineering, King's College London, London, U.K. His research interests include audio signal processing, room acoustics modeling and simulation, multichannel audio systems, psychoacoustics of spatial hearing, and microphone array processing. He is one of the core members of the EPSRC-funded Noise Futures Network.

Dr. Hacihabiboğlu is a member of the Audio Engineering Society (AES) and the European Acoustics Association (EAA).

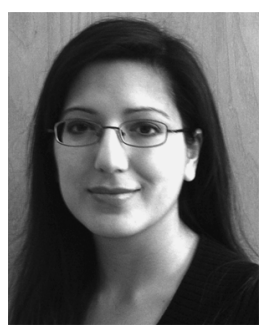

Banu Günel (S'97-M'00) was born in Antalya, Turkey, in 1977. She received the B.Sc. (Hons) degree in electrical and electronic engineering from the Middle East Technical University, Ankara, Turkey, in 2000, the M.Sc. degree in communication systems and signal processing from the University of Bristol, Bristol, U.K., in 2001, and the Ph.D. degree on audio and acoustical signal processing from the Queens University of Belfast, Belfast, U.K., in 2004.

Since 2004, she has been working as a Research Fellow in the Centre for Communication Systems Research (CCSR), University of Surrey, Guildford, U.K. Her main research interests are spatial audio, array signal processing, acoustic imaging, psychoacoustics, and communication acoustics.

Dr. Günel is a member of the European Acoustics Association (EAA) and the Audio Engineering Society (AES).

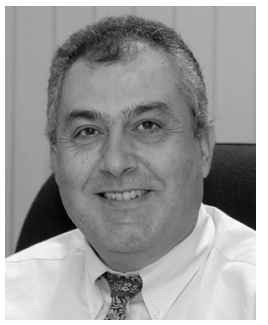

Ahmet M. Kondoz (M'91) was born in Cyprus. $\mathrm{He}$ received the B.Sc. (Hons) degree in engineering from the University of Greenwich, U.K., in 1983, the M.Sc. degree in telematics from the University of Essex, U.K., in 1984, and the Ph.D. degree in communication from the University of Surrey, Guildford, U.K., in 1986.

He became a Lecturer in 1988, a Reader in 1995, and then in 1996, a Professor in Multimedia Communication Systems and Deputy Director of Center for Communication Systems Research (CCSR), University of Surrey. He has over 250 publications, including two books on low-bit-rate speech coding and several book chapters, and seven patents. He has graduated more than $50 \mathrm{Ph} . \mathrm{D}$. students in the areas of speech/image and signal processing and wireless multimedia communications, and has been a consultant for major wireless media terminal developers and manufacturers. He is also a Director of Mulsys, Ltd., a University of Surrey spin-off company marketing the world's first secure GSM communication system through the GSM voice channel.

Prof. Kondoz has been awarded several prizes, the most significant of which are The Royal Television Societies Communications Innovation Award and The IEE Benefactors Premium Award. 